\title{
Cancer theory faces doubts
}

\section{A leading explanation for how disease migrates falls short on clinical evidence.}

\section{BY HEIDI LEDFORD}

I $t$ can't be easy to stand in front of hundreds of colleagues and tell them that ten years of research has led them in the wrong direction. But at the annual meeting of the American Association for Cancer Research (AACR) earlier this month, pathologist David Tarin did just that, by challenging a leading hypothesis on how cancers metastasize.

Tarin, from the University of California, San Diego, questioned the idea that cancer cells break free and migrate to new sites in the body by reverting to a state resembling the mobile cells in the developing embryo. Lab evidence for the hypothesis has raised hopes of some day blocking metastasis, which in $90 \%$ of cancer deaths is what ultimately kills the patient. But Tarin, along with some other cancer biologists, argues that no one has seen the process in action in human cancers.

Most cancers occur in the sheets of epithelial cells that line organs. Epithelial cells are normally immobile, but during embryonic development some start to produce proteins associated with motility and shut down production of proteins that glue the cells together. This transforms the cells into more mobile 'mesenchymal' cells that migrate to their correct locations in the embryo. If the same epithelial-to-mesenchymal transition (EMT) takes place in cancers, it could explain how tumour cells detach from their neighbours and enter the bloodstream to seed a new tumour (see 'Hypothesis under fire').

"It's a nice concept," says Pierre Savagner, a cancer researcher at the Montpellier Cancer Research Institute in France. "It makes things relatively simple to understand.”

Although controversial at first, the EMT hypothesis was bolstered by experiments in mice showing that activation of EMT led to metastasis. OSI Pharmaceuticals, based in Melville, New York, is already pursuing EMT inhibitors as possible cancer treatments, as are several academic labs.

"EMT is really becoming a popular subject," says Shoukat Dedhar, a cancer researcher at the British Columbia Cancer Research Centre in Vancouver and a self-avowed convert to the hypothesis. "There are more and more data coming out for EMT's role in metastasis."

Yet sceptics remain. Tarin, among others, is concerned that the hypothesis has achieved
For more on cancer and its prevention see: go.nature.com/rtvavt

\section{HYPOTHESIS UNDER FIRE}

Reactivation of a key developmental pathway could explain how tumour cells invade distant tissues.
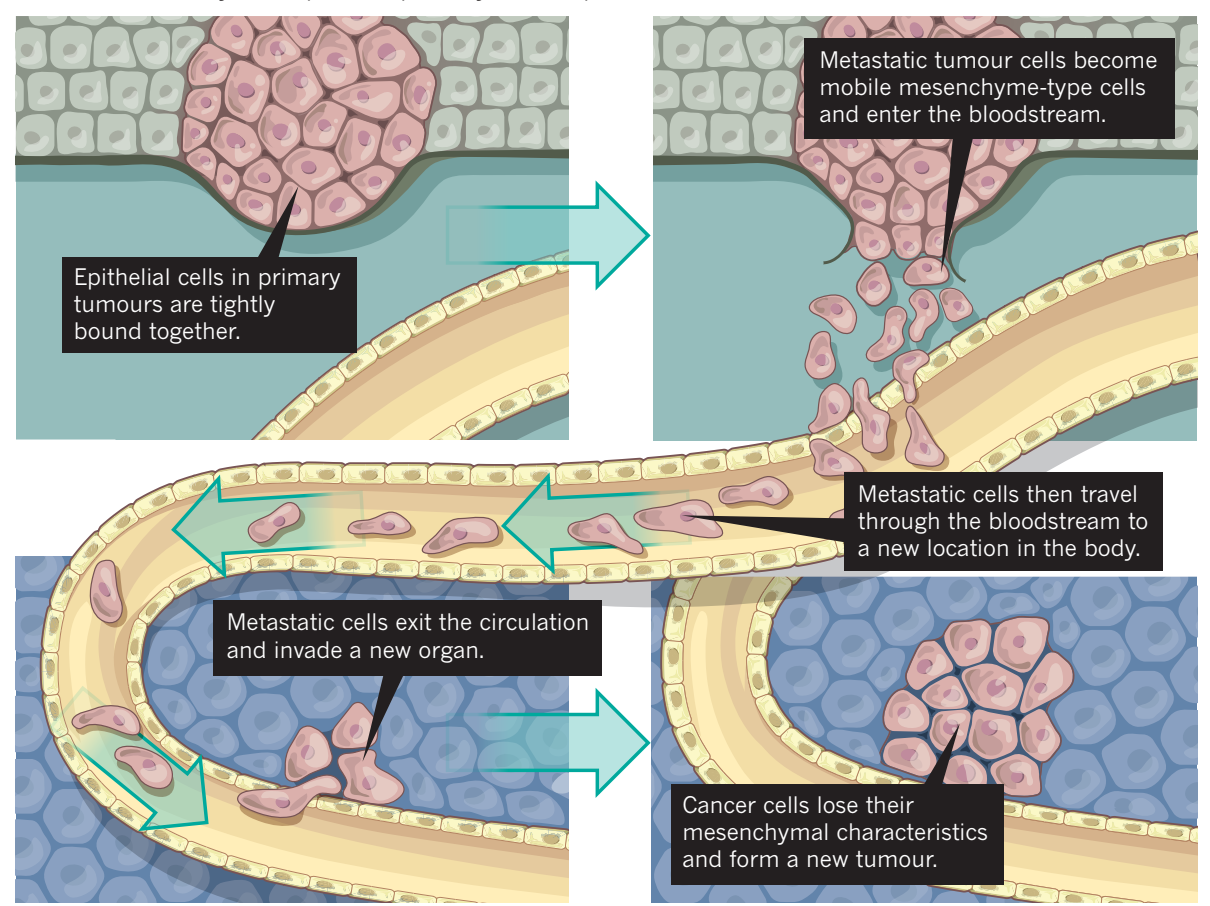

popularity on the basis of results from cultured cells and animal models, without convincing evidence of its importance in metastasis in humans. He argues that pathologists have combed through millions of tissue sections from tumours without seeing cells in transition.

\section{CONFLICTING IDEAS}

Champions of the EMT-metastasis hypothesis, including leading cancer biologist Robert Weinberg at the Whitehead Institute for Biomedical Research in Cambridge, Massachusetts, say that this may simply be because EMT is so transient - once a metastatic cell has invaded a new tissue, its mesenchymal features melt away. "When we examine a piece of tissue from a patient, we have just a snapshot of a moment," agrees cancer researcher Gianluigi Giannelli of the University of Bari Medical School in Italy. "We don't see the full movie."

Tarin dismisses this reasoning. "It's comparable to one telling you there are invisible aliens sitting in this room," he told the crowd at the AACR meeting, "but because you haven't used the right tools, you haven't seen them."

Others echo his concerns. Savagner points out that the proteins that might indicate EMT activation are also present during unrelated processes, such as programmed cell death.
"People really want these cells to do an EMT and they push the concept too far," he says.

Even Dedhar worries about overreaching claims. For example, many papers report results from cultured cells, he says, without confirming that the cells are metastatic in live animals.

The real picture, Tarin says, may not involve a change in cellular identity. Instead, he and others suggest that metastasis occurs when mutations in cancer cells compromise cell-cell adhesion. Others suggest that cells break off from the tumour in clumps and travel in packs.

Weinberg says a key experiment that could resolve the controversy would be to track individual cancer cells from the time they break off from a tumour to the point at which they colonize a new organ. Such an experiment would be technically challenging in humans, he says. "One can study the primary tumour and the cells it dispatches into the circulation," Weinberg says. "But finding out what happens to these circulating tumour cells after they become lodged is very difficult."

"Whether this process is mandatory for metastasis to occur is still unclear," says Isaiah Fidler, a cancer biologist at the M. D. Anderson Cancer Center in Houston, Texas. "But EMT is not to be dismissed. In cancer, we can't dismiss anything." 